\title{
Fatigue Crack Initiation and Early Growth in GLARE 3 Fiber-metal Laminate Subjected to Mixed Tensile and Bending Loading
}

A. Chlupová, J. Heger, A. Vašek

\begin{abstract}
A special open-hole sheet specimen of GLARE fiber-metal laminate was used to simulate mixed loading similar to the loading of a riveted hole in a real fuselage skin structure. The effect of cyclic tension and secondary bending loading was studied. The notch region was observed through a microscope in order to detect the first fatigue crack initiation during fatigue loading. The number of cycles prior to crack initiation was measured, and the crack growth rate in the surface layer of the laminate was evaluated. The specimens were subjected to fatigue damage investigation in the inner layers of the laminate after termination of the test. A significant effect of secondary bending on fatigue crack initiation and early crack growth was found. The experimental results are discussed in terms of local stress-strain conditions in the notch region evaluated by means of finite element calculation.
\end{abstract}

Keywords: laminate, composite, fatigue, mixed loading, finite element modeling.

\section{Notation}

$\begin{array}{ll}a & \text { crack length } \\ a_{\mathrm{f}} & \text { crack length at test termination } \\ e & \text { distance filler } \\ k & \text { bending factor } \\ N & \text { number of cycles } \\ N_{\text {in }} & \text { number of cycles prior to crack initiation } \\ R & \text { stress cycle ratio } \\ v & \text { fatigue crack growth rate } \\ \varepsilon_{\mathrm{pl}, 1} & \text { local plastic strain in the notch root of a specimen } \\ \sigma_{\text {bend }} & \text { induced bending stress } \\ \sigma_{\text {layer }} & \text { local stress in the individual metal layer of a laminate } \\ \sigma_{\text {max }} & \text { maximum applied tension stress }\end{array}$

\section{Introduction}

Fiber-metal laminates are a family of structural aerospace sheet materials, as shown in Vogelesang et al [1], that consist of thin metal sheets alternating with adhesive layers reinforced by high strength fibers. GLARE 3 fiber-metal laminate is composed of aluminium alloy layers and pre-impregnated layers of cross-plied high strength glass fibers in an epoxy-resin matrix. The cross-ply structure of the fibers makes this material suitable for applications subjected to bi-axial loading. GLARE 3 has been designed as an excellent material for the pressurized fuselage skin structure of large aircraft of the future. GLARE 3 laminate is currently being evaluated and tested in the Airbus A340 fuselage section, and is considered a promising candidate for the new $21^{\text {st }}$ century Airbus A3XX, as mentioned by Vlot et al [2].

Since riveting of large sheets is still the most common joining technology for a fuselage structure, rows of rivet holes are subjected to the highest attention. The holes locally induce stress concentration, and act as possible fatigue crack initiation sites. The pressurization of fuselage gives hoop stress, which is distributed over the joints. The load in the riveted lap joints is a combination of tension, bending, rivet load, clamp up and friction, as was shown by Müller [3]. In this study, attention is paid to the effect of mixed tension and bending loading on fatigue crack initiation and early growth in an open-hole sheet specimen.

\section{Experiments}

The GLARE 3 laminate used in the tests had been stacked with three $0.3 \mathrm{~mm}$ thick 2024-T3 aluminium alloy sheets alternating with two $0.25 \mathrm{~mm}$ thick epoxy resin layers reinforced by cross-plied continuous glass fibers $\left(0^{\circ} / 90^{\circ}\right)$ - see Figure 1. The laminate plate of total thickness $1.35 \mathrm{~mm}$ was cured in an autoclave at $120{ }^{\circ} \mathrm{C}$ under pressure 5 bar for 90 minutes.

A special asymmetric test configuration, as suggested by Nam et al [4], was adopted in order to introduce cyclical tension and bending in the sheet specimen loaded in a uniaxial fatigue machine. For this purpose both ends of the sheet specimen were flat bonded on in-axis sheet extensions made of the same material. An open hole of diameter $4.8 \mathrm{~mm}$ was drilled in the centre of the $40 \mathrm{~mm}$ wide and $100 \mathrm{~mm}$ long sheet specimens. The specimen surface in the vicinity of the central hole was mechanically polished in order to make fatigue cracks visible from their initiation. The specimens were cyclically loaded in the MTS 880 servo-hydraulic fatigue-testing machine, with the controlled load cycle of maximum stress $\sigma_{\text {max }}$ varying between $90 \mathrm{MPa}$ and $150 \mathrm{MPa}$ in individual tests. The stress cycle ratio was kept at $R=0.04$. The eccentricity of the specimen was preset by the different thickness of the filler inserted in the bonded lap joints. This enabled the bending moment to vary in different specimens. The geometry and dimensions of the specimen and the thickness of the fillers enabled the bending factor $k=\sigma_{\text {bend }} / \sigma_{\text {max }}$ to be adjusted in the interval between 0.5 and 1.5. Cyclic loading under pure tension $(k=0)$ was also applied. The specimen geometry is shown in Figure 1. 


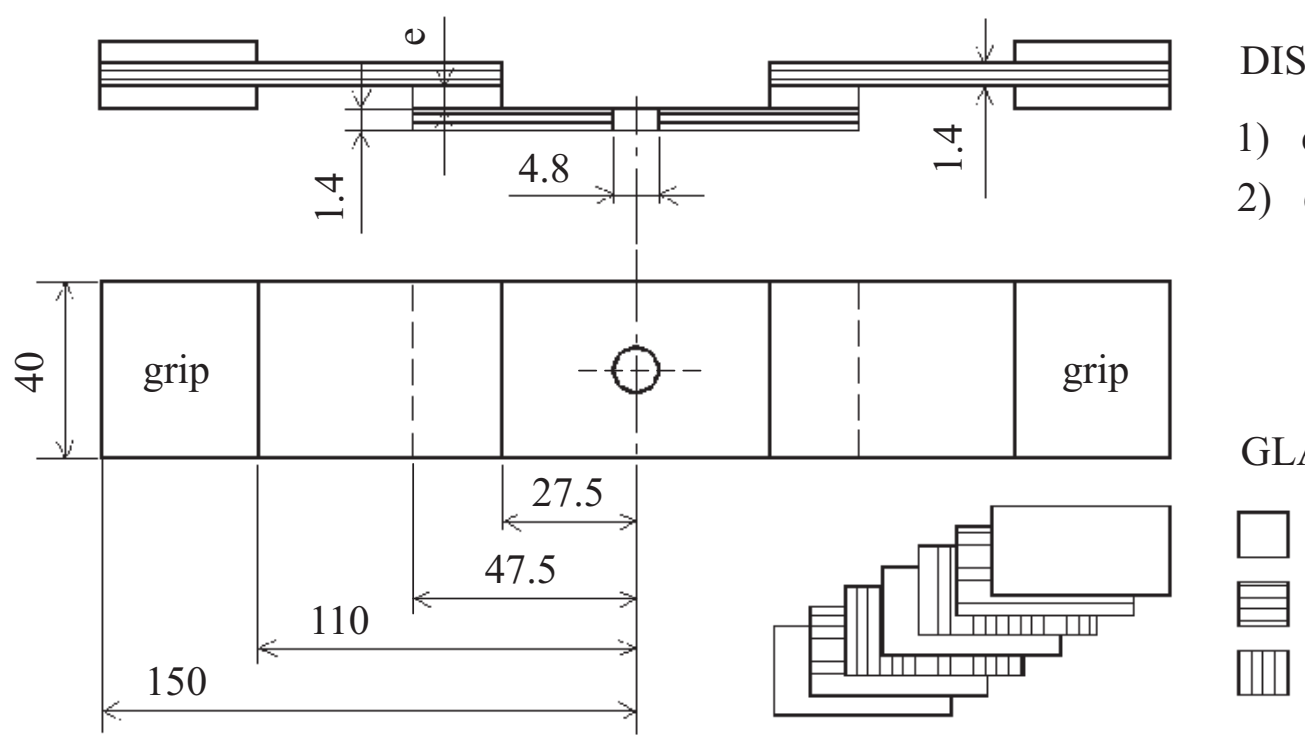

DISTANCE FILLER

1) $\mathrm{e}=0 \mathrm{~mm}$

2) $\mathrm{e}=1 \mathrm{~mm}$

GLARE $3(3 / 2)$

$\mathrm{Al}(2024-\mathrm{T} 3), 0.3 \mathrm{~mm}$

Prepreg $\left(0^{\circ}\right), 0.125 \mathrm{~mm}$

Prepreg $\left(90^{\circ}\right), 0.125 \mathrm{~mm}$

Fig. 1: Specimen geometry and laminate composition of GLARE 3

We performed finite element stress and strain modelling in GLARE 3 laminate sheet subjected to mixed tensile and secondary bending. Each prepreg layer was modelled by two element layers, and each aluminium layer was modelled by four element layers. A denser mesh of finite elements was used in the vicinity of the rivet hole (Figure 2) to give a true picture of the real stress and strain concentration effect. Meshing was performed by the "mapped meshing" technique, using six sided solid elements exclusively. Even if double symmetry were used, the total number of elements would exceed 20,000 and the total number of nodes 23,000. A special FE procedure was developed in order to take into account both the stress-strain non-linear response in the metal layers and the geometrical non-linearity of the large specimen deflection due to the bending moment, as suggested by Heger [5]. The ANSYS finite element system was adopted for the solution.

The notch region was observed through a light microscope in order to detect the initiation of the first fatigue crack and to measure the number of cycles prior to crack initiation

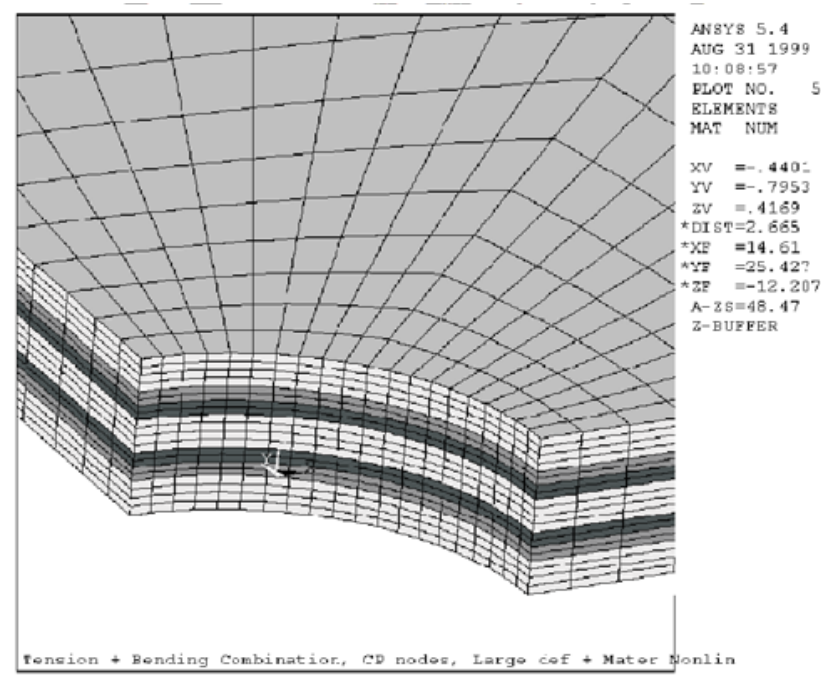

Fig. 2: Detail of the FE mesh in the vicinity of the rivet hole during loading. The growth of the fatigue cracks was periodically measured after initiation on the specimen surface with the aim of evaluating the crack growth rate in the metal layer surface of the laminate. The loading was terminated when the surface crack, measured from the hole edge, was about $10 \mathrm{~mm}$ in length. The specimens were subjected to a destructive investigation of the fatigue damage in the inner layer of the laminate after termination of the test. The surface metal layers were electrochemically milled off and the adhesive layers with glass fibers were removed mechanically in the procedure. Then the disclosed surface of the inner metal layer was electrochemically polished and the fatigue cracks in this originally hidden layer could be revealed.

\section{Results}

First fatigue cracks were initiated in the surface metal layer that was under the greatest bending stress. The number of cycles prior to crack initiation versus maximum applied stress $\sigma_{\max }$ is plotted in Figure 3. The different positions of the

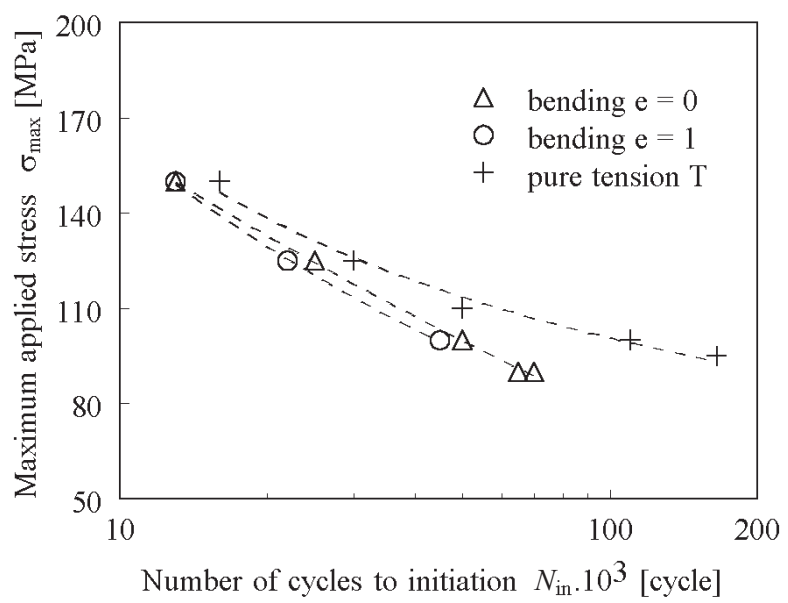

Fig. 3: Graph of the number of cycles prior to initiation $N_{\text {in }}$ versus maximum applied stress $\sigma_{\max }$ for different thickness of the distance filler 
curves confirm that the additional bending stress reduces the initiation period in GLARE 3 laminate. The effect is more obvious at low cyclic stress when the bending/tension rate $k$ is higher.

The crack growth of surface cracks was measured during the experimental loading. Figure 4 shows graphs of the crack length $a$, measured from the notch root dependent on the number of cycles $N$. The experimental data in the figure were acquired in tests with $\sigma_{\max }=125 \mathrm{MPa}$ and with different bending stress $\sigma_{\text {bend }}=0,80$ and $137 \mathrm{MPa}(k=0,0.64$ and 1.1). As shown in Figure 4, the crack growth data can fit a linear dependence. This implies that crack growth rate $v=\mathrm{d} a / \mathrm{d} N$ was approximately constant in all performed tests.

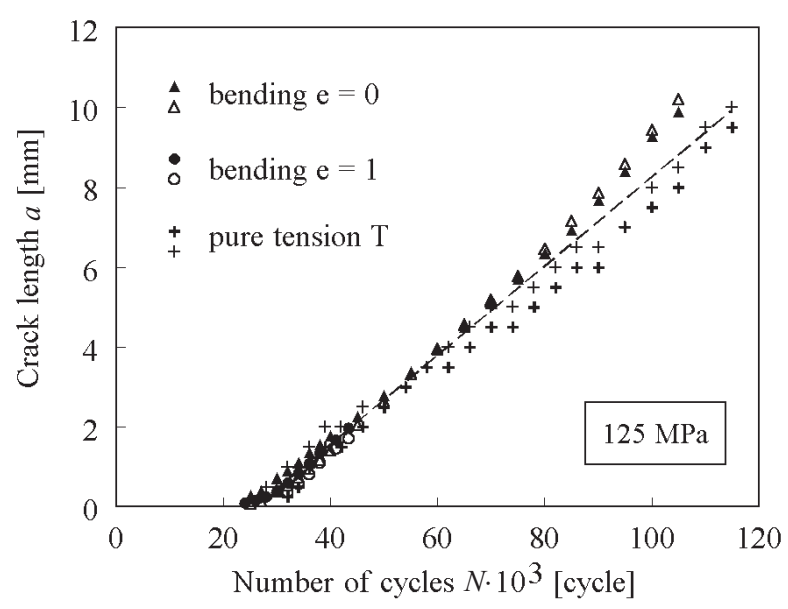

Fig. 4: Dependence of crack length on the number of cycles for the applied stress $\sigma_{\max }=125 \mathrm{MPa}$ (at different bending factor $k$ )

The length of the cracks in the inner metal layer was measured after termination of the test. A significant difference between final crack lengths was found comparing cracks in the individual metal layers. The difference was affected by the bending factor $k$. Higher $\mathrm{k}$ resulted in more different crack lengths on opposite surfaces of the laminate plate. Figure 5 shows an example of crack lengths in all three metal layers of laminate specimens subjected to $\sigma_{\max }=125 \mathrm{MPa}$ with varying bending stress $\sigma_{\text {bend }}=0,80$ and $137 \mathrm{MPa}(k=0,0.64$ and 1.1). The relative crack length

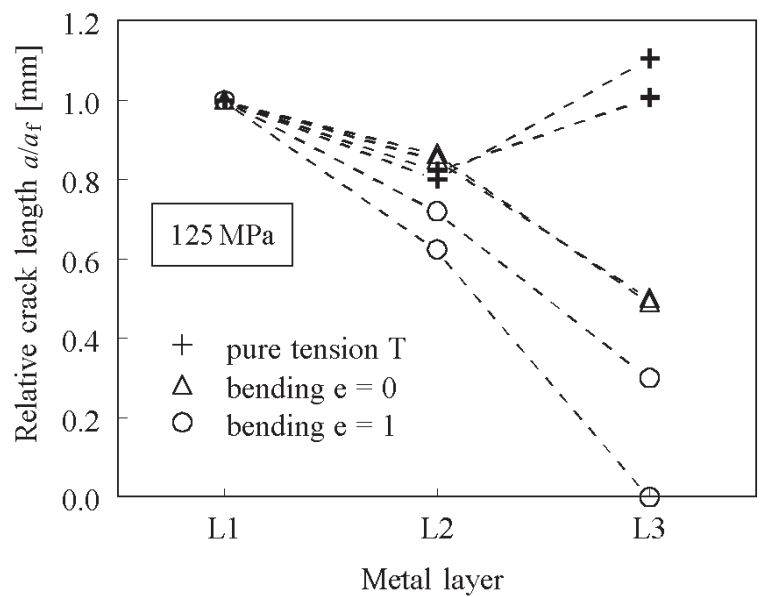

Fig. 5: Relative crack length in the individual metal layers for the applied stress $\sigma_{\max }=125 \mathrm{MPa}$ and various distance fillers $a / a_{\mathrm{f}}$ (where $a_{\mathrm{f}}$ is the crack length in the upper layer at test termination) was plotted for better comparison.

\section{Discussion}

First fatigue cracks were found in the surface metal layer that was subjected to the maximum resulting stress. Cracks in the inner metal layer occurred later, and the latest fatigue cracks were initiated in the surface layer that was subjected to the minimum resulting stress.

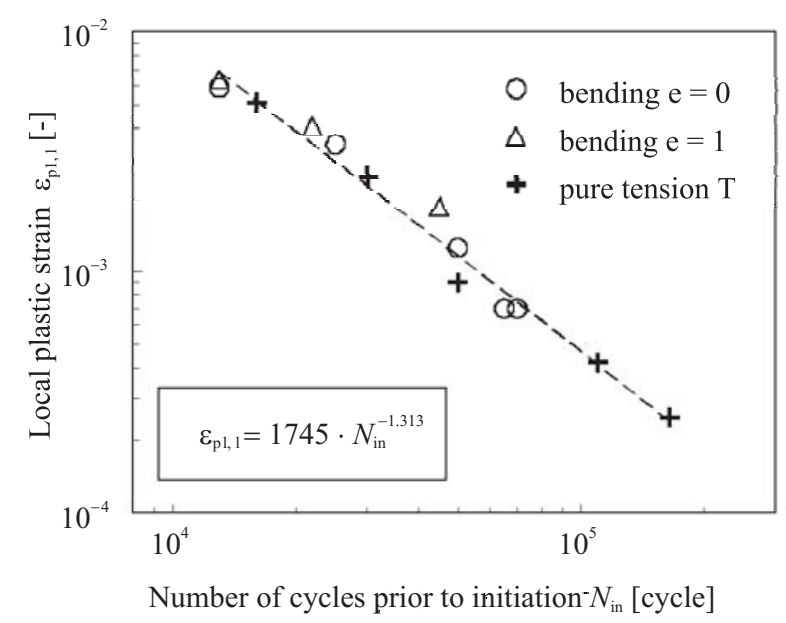

Fig. 6: Graph of the experimental data of $N_{\text {in }}$ from all tests versus local plastic strain $\varepsilon_{\mathrm{pl}, 1}$ in the notch root

In accordance with a recent investigation of fatigue crack initiation mechanisms described in Polák [6], the local plastic strain $\varepsilon_{\mathrm{pl}, 1}$ in the notch root was calculated by the finite element method in order to find a parameter $N_{\text {in }}$ determining the number of cycles necessary for the initiation of fatigue cracks. The experimental data of $N_{\text {in }}$ from all tests vs. local plastic strain are plotted in Figure 6, where a power-law relation fits the curve:

$$
\varepsilon_{\mathrm{pl}, 1}=1745 \cdot N_{\mathrm{in}}^{-1.313} \text {. }
$$

Although the experimental data in Figure 6 were taken only from the surface layers, the relation (1) can be used for predicting $N_{\text {in }}$ also in the inner layer when the local plastic strain in the layer $\varepsilon_{\mathrm{pl}, \mathrm{l}}$ is known.

The investigation revealed that the crack growth rate in the performed tests was approximately constant. The crack growth in this kind of material is determined predominantly by an effect of the crack bridging fibers, which restrain the crack opening and thus decrease the stress intensity factor. The crack bridging effect increases with increasing crack length, due to an increase in the number of fibers affected in longer cracks. Depending on the laminate structure, the bridging effect can reduce the crack growth rate expected for monolithic metallic materials and can even cause a decrease in the crack growth rate with increasing crack length (e.g., GLARE 2 in Vašek [7]). In the special case of the GLARE 3 laminate the crack growth rate was found to be constant and independent of the crack length. Assuming that the accelerating effect of increasing crack length is compensated by the retarding effect of the crack bridging fibers, the stress intensity factor and the crack growth rate can be determined by a local stress acting in a separate metal layer. Figure 7 shows 
the dependence of the crack growth rate $\mathrm{v}$ on the stress in the surface metal layer calculated by means of finite element modeling. A semi-logarithmic relation

$$
v=7.17 \cdot 10^{-10} \exp \frac{\sigma_{\text {layer }}}{10.8}
$$

was derived that fits the experimental results. Relation (2) holds for $v$ in $\mu \mathrm{m}$ and $\sigma_{\text {layer }}$ in MPa and can also be used for predicting the crack growth rate and/or residual life in all metal layers of the laminate when $\sigma_{\text {layer }}$ is known.

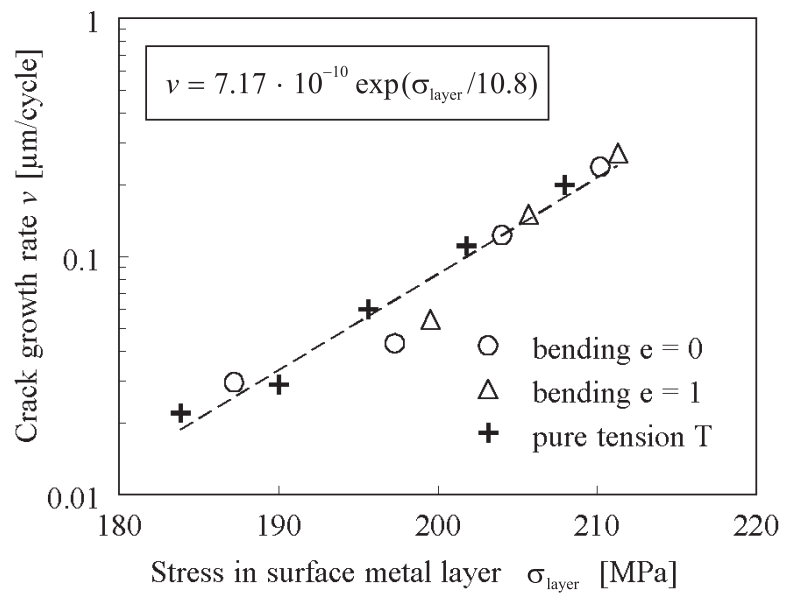

Fig. 7: Dependence of crack growth rate on the stress in a surface metal layer $\sigma_{\text {layer }}$ for different values of the distance filler

\section{Conclusions}

An experimental investigation of fatigue crack initiation and early growth in GLARE 3 fiber metal laminate plates subjected to mixed tensile-bending loading was analysed by means of finite element modelling of local stress and strain in the individual laminate layers. The most significant results are as follows:

- An asymmetric lap-joint tension specimen was adopted in order to produce a mixed tension-bending cyclic loading.

- The first fatigue cracks were found in the upper surface layer subjected to the maximum cyclic resulting stress. Cracks in the central layer initiated later and the latest cracks initiated in the opposite surface layer under minimum resulting stress. The number of cycles necessary for crack growth through the laminate thickness increased with the higher rate $\sigma_{\text {bend }} / \sigma_{\text {max }}$. The through-thickness crack was separated by intact fibers bridging the crack.

- The additional cyclic bending loading shortened the crack initiation period and increased the crack growth rate in comparison with pure tensile cyclic loading.

- The number of cycles necessary for fatigue crack initiation in metal layers of the laminate was determined by the local plastic strain at the notch edge in the individual layers. The received relation can be used to predict the number of cycles prior to the initiation in outer or inner metal layers.

- The surface crack growth rate was found to be constant and independent of the crack length. Knowledge of the stress in individual metal layers enabled the crack growth rate also to be evaluated in the inner metal layers.

\section{Acknowledgment}

This work was financially supported by the Academy of Sciences of the Czech Republic (Grant No. S/204/1001), Grant Agency of the Academy of Sciences of the Czech Republic (Grant No. C/204/1104), Grant Agency of the Czech Republic (Grant No. 106/01/1464, 106/01/1164, 106/99/0728) and by the CEZ Research Project: 322/98: 262100001 .

\section{References}

[1] Vogelesang, L. B., Gunning, J. W.: Materials and Design. Vol. 7, 1986, pp. 278-300

[2] Vlot, A., Vogelesang, L. B., de Vries, T. J.: Towards application of fibre metal laminates in large aircraft. http://www.glareconference.com/glare.htm, 2001

[3] Müller, R. P. G.: An Experimental and Analytical Investigation on the Fatigue Behaviour of Fuselage Riveted Lap Joints. PhD thesis, Delft University of Technology, 1995

[4] Nam, K. W., et al: Fatigue Life and Penetration Behaviour of a Surface-Cracked Plate under Combined Tension and Bending. Fatigue and Fracture of Engineering Materials and Structures, Vol. 17, 1994, pp. 873-882

[5] Heger, J.: Combined Tensile-Bending Load Simulation of the Notched Thick Wall Multi-Layer Laminate Composite Used for Aircraft Body Building. Ninth International ANSYS Users Conference and Exhibition, Pittsburgh, PA, IV/2, 2000

[6] Polák, J.: Cyclic Plasticity and Low Cycle Fatigue Life of Metals. Academia Praha, 1991

[7] Vašek, A., Prášilová, A.: Retarding effect of reinforcing fibres on early crack growth in fatigued notched laminate GLARE 2. Engineering Mechanics, Vol. 5, 1998, pp. 219-223

Ing. Alice Chlupová

phone: +420541636344

fax: +420541218657

e-mail: prasil@ipm.cz

Institute of Physics of Materials

Academy of Sciences

Žižkova 22, 61662 Brno, Czech Republic

RNDr. Alois Vašek, CSc.

phone: +420547125328

fax: +420547125310

e-mail:vasek@bibus.cz

Institute of Physics of Materials

Academy of Sciences

Žižkova 22, 61662 Brno, Czech Republic temporary address: BIBUS s.r.o.

Vídeňská 125, 63927 Brno, Czech Republic

Ing. Jaromír Heger, CSc.

phone: +420541142888

fax: +420541218657

e-mail: heger@umt.fme.vutbr.cz

Institute of Solid Mechanics

Technical University

Technická 2, 61669 Brno, Czech Republic 